\title{
Towards the sensitivity for the radar scattering technique to probe neutrino induced particle cascades in ice: The radar cross-section
}

\section{Krijn D. de Vries*, Nick van Eijndhoven, Simona Toscano}

Vrije Universiteit Brussel, Dienst ELEM, IIHE, Pleinlaan 2, 1050, Brussel, Belgium

E-mail: krijndevries@gmail.com

\section{Aongus O'Murchadha}

Dept. of Physics and Wisconsin IceCube Particle Astrophysics Center, University of Wisconsin, Madison, WI 53706, USA

\section{Olaf Scholten}

University of Groningen, KVI Center for Advanced Radiation Technology, 9747 AA Groningen, The Netherlands

Vrije Universiteit Brussel, Dienst ELEM, IIHE, Pleinlaan 2, 1050, Brussel, Belgium

\begin{abstract}
We discuss the feasibility of the radar detection technique to probe high-energy neutrino-induced particle cascades in ice. The properties of the plasma which is created when a high-energy neutrino-induced particle cascade traverses the medium are modeled in detail for random scattering geometries. This allows us to model the radar scattering cross section for generic cases, where previously our results were based on an empirical thin-wire approximation.
\end{abstract}

35th International Cosmic Ray Conference -ICRC2017-

10-20 July, 2017

Bexco, Busan, Korea

\footnotetext{
* Speaker.
} 


\section{Introduction}

Recently, we investigated the radar detection technique to probe high-energy particle cascades in ice $[1,2,3]$. The main goal of these investigations is to cover the currently exisiting energy gap for the detection of high-energy cosmic neutrinos. This energy gap is found between several PeV, where IceCube runs low in statistics [4], and a few $\mathrm{EeV}$, where the currently operating Askaryan radio detectors start to become sensitive $[5,6,7]$.

In [1], we considered different types of plasmas created by a high-energy neutrino induced particle cascade propagating in ice. Here, it was shown that both the free electron, as well as the free proton, ionization plasmas should allow for an efficient radar scatter. This scattering efficiency is consequently reflected in the radar cross-section, which was taken from an emperically derived thin-wire approximation [8]. In these proceedings, we derive the radar cross-section, based on the details of the over-dense plasma profile. This allows us to calculate the scattering process for a random geometry. Furthermore, we briefly discuss several up to now unknown parameters, such as the lifetime and the free charge collision rate, which are crucial to obtain efficient scattering.

Since these parameters are currently unknown, they have to be determined experimentally. Therefore, recently two radar scattering experiments have been performed at the Telescope Array Electron Light Source facility [9]. Data analysis of these experiments is currently still ongoing, but up to now no radar scatter has been observed. Using the model presented in this work, we are also able to estimate the detector efficiency for a simplified set-up. Once folded with the neutrino interaction probability, this can be used to obtain the sensitivity to the cosmic neutrino flux, which will be presented in a future work.

\section{The plasma}

A relativistic charge will deposit roughly $2 \mathrm{MeV} \mathrm{g}^{-1} \mathrm{~cm}^{-2}$ of its energy by ionizing the medium in which it travels, ice in our situation. This energy deposit only depends logarithmically on the original energy of the particle, and hence throughout this article we consider this to be constant. Consequently, if a high-energy particle cascade traverses a medium like ice, we can consider three different plasmas. First there is the high-energy cascade front itself, which at shower maximum typically consists of $N_{\max } \approx E_{p} /\left(10 E_{c}\right)$ particles, mainly electrons and positrons. Here $E_{p}$ is the energy of the cascade inducing particle, and $E_{c}$ denotes the critical energy of an electron in the medium in which the cascade propagates. The factor 10 is included since roughly $90 \%$ of the cascade energy is contained in high-energy photons close to the propagation axis. Since the cascade ionizes the medium, we also find a free charge plasma which is left behind when the cascade passes by. Experimental results from the 1970s show evidence for both a free electron plasma, as well as a free proton plasma, which were found when irradiating a block of ice with either X-rays or $\mathrm{MeV}$ electrons $[10,11]$. Here it was also shown that the plasma lifetime strongly depends on the ice temperature. The free electron plasma was found to have a lifetime below $1 \mathrm{~ns}$ at temperatures close to $0^{\circ} \mathrm{C}$, up to several tens of nanoseconds for temperatures around $-50^{\circ} \mathrm{C}$. The free proton plasma was found to have a longer lifetime ranging from roughly $10 \mathrm{~ns}$ up to a microsecond over the same temperature range. 


\section{Radio wave scattering}

To determine the radio wave scattering, we need to consider two different plasma regions, which are distinguished by the free charge density. When the plasma is dense enough, so-called over-dense scattering occurs over the full surface area of the plasma. This condition is met when the radar frequency at which the plasma is probed falls below the plasma frequency, $v_{\text {radar }}<v_{\text {plasma }}$, where $v_{\text {plasma }}=8980 \sqrt{\frac{m_{e}}{m_{\text {plasma }}} n_{e}}$, with the plasma number density given by $n_{e}\left[\mathrm{~cm}^{-3}\right]$. In case this condition is not fulfilled, the plasma is not seen as a collective volume and the scattering occurs off the individual electrons by means of Thomson scattering, with a scattering cross-section equal to,

$$
\sigma_{T}=\left(\frac{m_{e}}{m_{\text {plasma }}}\right)^{2} 0.665 \cdot 10^{-28} \mathrm{~m}^{2},
$$

where $m_{e}$ denotes the electron mass and $m_{\text {plasma }}$ the mass of the plasma constituent.

\subsection{Under-dense scattering}

The total under-dense radar cross-section is now obtained by taking into account the phase lag between the reflected signals of the individual electrons. This is given by,

$$
\sigma_{u d}=\sum_{i=1}^{N} \sigma_{T} \cos (k x)
$$

where $k=\frac{2 \pi}{\lambda_{d}}$, and $x=\left|\vec{x}_{t}-\vec{x}_{i}\right|+\left|\vec{x}_{r}-\vec{x}_{i}\right|$, where $\vec{x}_{t}$ denotes the location of the transmit antenna, $\vec{x}_{r}$ gives the position of the receiver antenna, $\vec{x}_{i}$ denotes the free charge position, and $\lambda_{\text {radar }}$ denotes the wavelength of the incoming radio wave.

\subsection{Over-dense scattering}

If the radar frequency is below the plasma frequency, $v_{\text {radar }}<v_{\text {plasma }}$, the incoming wave scatters over-dense. In this situation, instead of probing the individual charges, the incoming wave is able to scatter over the entire plasma surface. Where previously we considered a thin-wire approximation to determine the effective scattering surface [8], in these proceedings we closely follow the approach presented in [2], where we derive the effective scattering cross-section for a simplified geometry. To do so, we first model the free charge plasma. By assuming that the highenergy particles in the cascade deposit on average $2 \mathrm{MeV} \mathrm{g}^{-1} \mathrm{~cm}^{-2}$ of ionization energy, we show in Fig. 1 the ionization plasma density as a function of the cascade depth. The details of the particle cascade itself are outlined in [2]. While a simplified geometry was considered in [2], we consider a more general geometry where scattering occurs over the projected surface area in the polarization plane of the incoming wave. The projection effects will be accounted for by the geometry factor $f_{\text {geom }}$,

$$
f_{\text {geom }}=\left(1-\vec{e}_{t c} \cdot \vec{e}_{c}\right)\left(\vec{e}_{t} \cdot \vec{e}_{c}\right) f_{\text {diff }} .
$$

Here $\vec{e}_{t c}$ denotes the unit vector pointing from the transmitter to the cascade, $\vec{e}_{c}$ denotes the unit vector along the cascade direction, and $\vec{e}_{t}$ denotes the polarization of the transmitted signal in the cascade plane. Finally we also included the diffraction factor $f_{\text {diff }}$, which is outlined in more detail in [2]. Since the wavelength of the emitted wave is typically of the order of the dimensions of the 


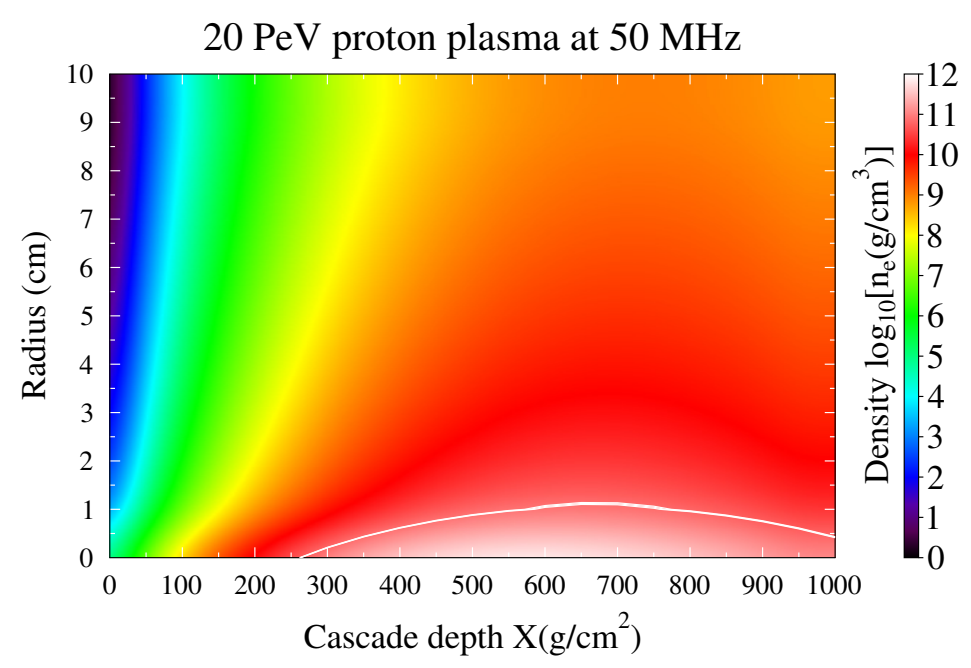

Figure 1: The free charge proton plasma for a $20 \mathrm{PeV}$ electromagnetic cascade in ice. The white line indicates the over-dense region in case the plasma is probed at a frequency of $v_{\text {radar }}=50 \mathrm{MHz}$. Figure taken from [2].

cascade, we do not expect specular reflection, but rather a Fraunhofer single-slit diffraction pattern, which introduces a factor,

$$
f_{\text {diff }}=\frac{I(\alpha)}{<I(\alpha)>}
$$

where $I(\alpha)=\operatorname{sinc}^{2}(\beta)$, with $\beta=(\pi L / \lambda) \sin \alpha$. The size of the over-dense plasma region is given by $L$, and $\alpha$ is the angle relative to the angle of total internal reflection in the cascade plane.

Since we do not have a uniform plasma, we consider the scattering to occur over layers of approximately equal density. Following [2], the fraction of an incoming wave which is seen and consequently reflected by a plasma layer of thickness $\Delta x$ can be deduced by considering the skin depth, $\delta=c /\left(2 v_{\text {plasma }}\right)$, and is given by,

$$
f_{\text {scatt }}^{i}=\left(1-f_{\text {scatt }}^{i-1}\right)\left(1-e^{-(\Delta x)^{i} / \delta_{p}^{i}}\right)
$$

Note that we implicitly assume that all power that is reaching the plasma is reflected in case $v_{\text {radar }}<$ $v_{\text {plasma }}$, and that the actual absorption is low. To include the actual absorption is hard, since it depends on the free charge collisional damping [12]. This is currently the largest unknown in our model, where for example in air the absorption was shown to be too large to obtain an efficient scatter [13]. Therefore, we include an overall efficiency factor $\eta$ to account for any of such losses. This factor will not only depend on the absorption, but also on the, so-far unknown, lifetime of the free charge plasma, and therefore has to be determined experimentally. The total over-dense radar scattering cross-section is now obtained as,

$$
\sigma_{o d}=\eta \sum_{i} A^{i} \times f_{\text {geom }} \times f_{\text {scatt }}^{i},
$$

where $A^{i}$ is the area of the $\mathrm{i}$-th layer of equal density. 


\section{The scattering efficiency for a simplified radar set-up}

Using the model described in the previous section, we are now able to calculate the radar cross-section $\sigma_{\text {radar }}=\sigma_{u d}+\sigma_{o d}$. To determine the scattering efficiency, we can consider a bi-static radar configuration. For this configuration, the observed scattering power is given by,

$$
P_{\text {scatt }}=P_{\text {emit }} \frac{G}{4 \pi} \frac{\sigma_{\text {radar }}}{\left|\vec{x}_{t}-\vec{x}_{c}\right|^{2}} \frac{A_{\text {eff }}}{\left|\vec{x}_{c}-\vec{x}_{r}\right|^{2}},
$$

where the transmit power is given by $P_{\text {emit }}, G$ denotes the directional gain of the transmitted wave, $\vec{x}_{c}$ denotes the position of the cascade, and $A_{e f f} \approx \lambda_{r}^{2}$ is the effective area of the receiver antenna which is assumed to scale quadratically with the wavelength of the emitted radar signal $\lambda_{r}$. Since we now are able to calculate the scattered power for a random geometry, we can calculate the efficiency for a simplified radar set-up. This is done by considering the layout given in Fig. 2. We consider an array of 5 transmit antennas and 16 reciever antennas on a $3 \times 3 \mathrm{~km}$ grid. Each transmit antenna is surrounded by 4 reciever antennas positioned $500 \mathrm{~m}$ to the North $(+\mathrm{y})$, East $(+\mathrm{x})$, South $(-\mathrm{y})$, and West (-x). We assume that the transmit antennas emit $1 \mathrm{~kW}$ in both horizontal (x and y)polarizations as well as the vertical z-polarization, and assume that the recievers are able to recieve in all polarizations. To obtain the efficiency of such a set-up, we generated a semi-isotropic set of

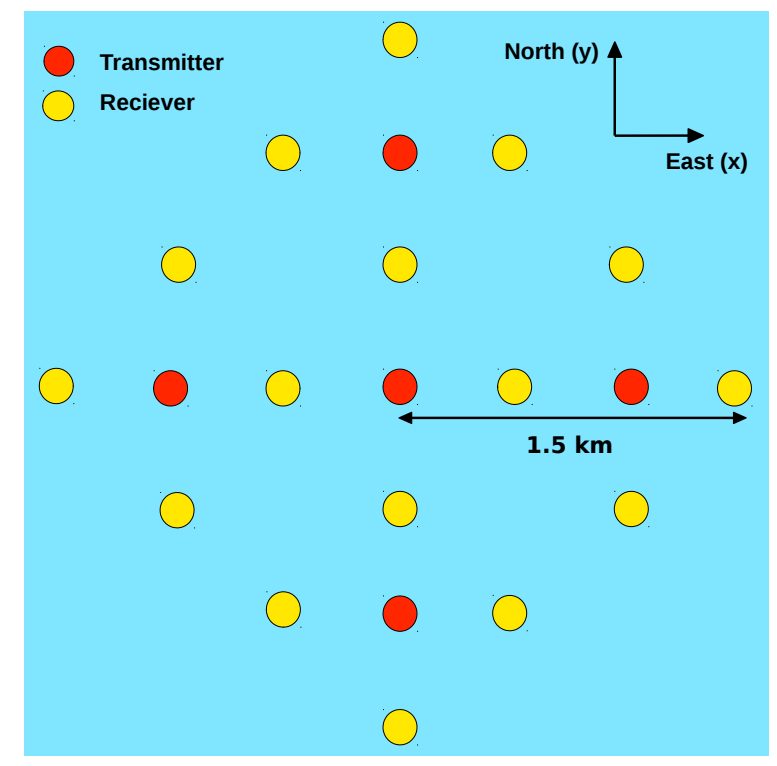

Figure 2: The non-optimized radar detection set-up used to calculate the radar detection efficiency presented in Fig. 3.

particle cascades with energies in the $\mathrm{PeV}$ to $\mathrm{EeV}$ region, within a cylinder of radius $2 \mathrm{~km}$, and depth $2.8 \mathrm{~km}$ corresponding to the South Pole ice depth. Due to the Earth absorption effect, we do not expect any upgoing neutrino induced particle cascades at the investigated energy range, hence we only consider a zenith range between $0^{\circ}-90^{\circ}$ degrees. Furthermore, we fix $\eta=0.1$, and label an event as detected if one of the reciever antennas detects a signal above the background which is given by thermal noise $P_{n o i s e}=k_{b} T_{n}$. Here we take $T_{n}=325 \mathrm{~K}$ as obtained at the South Pole by the ARA collaboration [5]. 
The efficiency of the detector is shown in Fig. 3. Here we show the results for the scattering off a free electron plasma with a lifetime of $\tau_{e}=20 \mathrm{~ns}$, probed at $v_{\text {radar }}=450 \mathrm{MHz}$, and the scattering off a free proton plasma with a longer lifetime of $\tau_{p}=1 \mu \mathrm{s}$. It follows that the efficiency for scattering off the free electron plasma is approximately $1 \%$, where for the free proton plasma this ranges from $10-50 \%$ for PeV-EeV energies, respectively.

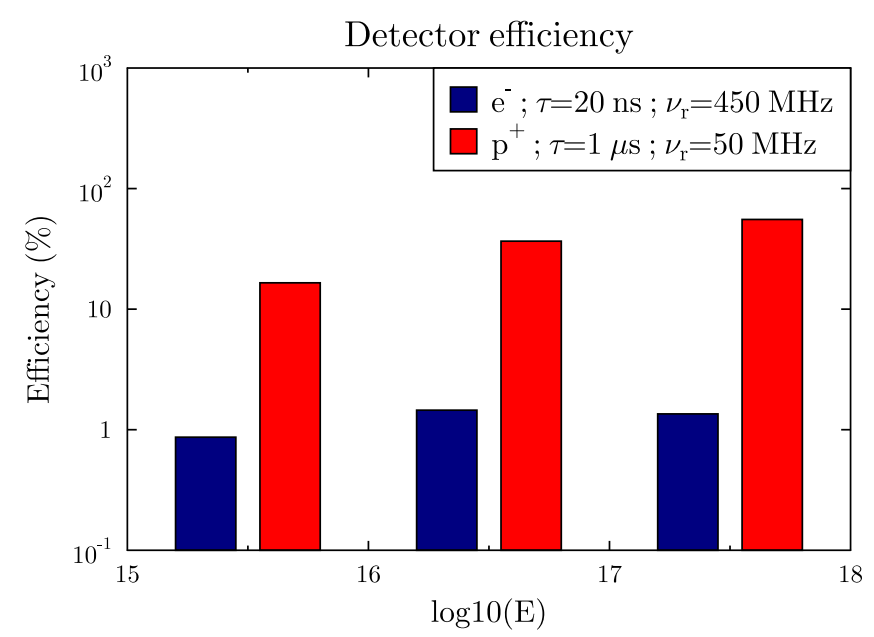

Figure 3: The scattering efficiency for the detector layout presented in Fig. 2. The efficiency is shown for scattering off a free electron plasma with a lifetime of $\tau_{e}=20 \mathrm{~ns}$, probed at $v_{\text {radar }}=450 \mathrm{MHz}$, and a free proton plasma with a longer lifetime of $\tau_{p}=1 \mu \mathrm{s}$, probed at $v_{\text {radar }}=50 \mathrm{MHz}$.

\section{Outlook}

We presented a model to obtain the radar scattering cross-section for a high energy particle cascade in ice by taking into account the projected geometry, as well as the amount of power which is seen by the cascade. As a first application of this model, we show the scattering efficiency for a simplified radar set-up. For scattering off the free electron plasma, we find an efficiency of roughly $1 \%$, where the free proton plasma shows an efficiency between $10-50 \%$ for energies ranging from $\mathrm{PeV}$ to EeV for a generation volume of $35 \mathrm{~km}^{3}$. It should be noted that for this calculation, we assume a rather progressive efficiency factor $\eta=0.1$ to cover the unknown plasma parameters such as its lifetime, and the free charge collision rate.

This calculation will be extended by considering a realistic neutrino flux, and the neutrino interaction probability within the detector volume, which should allow us to determine the sensitivity to the expected cosmic neutrino flux in the PeV-EeV range for the presented set-up .

\section{References}

[1] K.D. de Vries, K. Hanson, T. Meures, Astropart. Phys. 60 (2015) 25-31

[2] K.D. de Vries et al., EPJ Web Conf. 135 (2017) 05006 
[3] K.D. de Vries et al., PoS (ICRC2015) 1168 (2015)

[4] IceCube Collaboration, Science 342 (2013) 1242856

[5] P. Allison et al., Astropart. Phys. 35 (2012) 457-477

[6] A. Nelles et al., ARIANNA collaboration, EPJ Web Conf. 135 (2017) 05002

[7] P.W. Gorham et al., Phys. Rev. Lett. 103 (2009) 051103

[8] J.W. Crispin Jr., A.L. Maffett,Proc. Roy. Soc. A 53 (1965) 833

[9] T.Shibata et al., Nucl. Instr. Meth. A 597 (2009) 61-66

[10] J.B. Verberne et al., Nature 272 (1978) 343-344

[11] M. Kunst, J.M. Warman, Nature 288 (1980) 465-467

[12] J. Stasielak et al., Astropart.Phys. 73 (2016) 14-27

[13] R.U. Abbasi et al., Astropart.Phys. 87 (2017) 1-17 